\title{
PROBLEMAS E DISTINÇÕES ENTRE MÉTODOS DE AVALIAÇÃO DA CONDIÇÃO GERAL DE ÁRVORES URBANAS
}

\author{
PROBLEMS AND DISTINCTIONS AMONG METHODS FOR ASSESSMENT OF THE \\ GENERAL CONDITION OF URBAN TRESS
}

\author{
Rogério Bobrowski ${ }^{1}$
}

\section{RESUMO}

Os protocolos de avaliação da condição geral de árvores urbanas servem como roteiros metodológicos para direcionar a avaliação de uma árvore, principalmente quando não se dispõe de instrumentos eletrônicos para a inspeção ou quando se tem um grande volume de árvores para avaliar. Cada protocolo apresenta uma abordagem específica para a avaliação, mais abrangente ou mais específico, porém sempre apresentando um grau de subjetividade. Nesta pesquisa, três protocolos foram utilizados na avaliação da condição de quarenta árvores (dez de cada espécie florestal) existentes em duas áreas verdes da cidade de Irati-PR. Por meio do Teste de Friedman constatou-se diferença significativa entre os protocolos utilizados ( $\chi^{2}$ Fried $=69,194 ; \mathrm{GL}=2 ; p=9,434^{-16}$ ) para a valoração atribuída a cada árvore. Entretanto, para melhor análise visual da condição geral de árvores urbanas, os protocolos devem contemplar a introdução de informações sobre defeitos estruturais, condição do ancoramento da árvore ao solo, existência de pragas ou doenças e o grau de risco oferecido. Estas adaptações se tornam necessárias e estratégicas para direcionar a atenção do avaliador para as principais regiões de avaliação e para a redução da subjetividade.

Palavras-chave: Avaliação visual, Vigor, Risco, Arboricultura.

\begin{abstract}
The urban tree risk assessment forms serve as a methodological routine to guide a tree evaluation mainly when there are no electronic instruments for inspection or when there are a lot of trees to assess. Every assessment form shows a specific approach to assess the tree, more inclusive or more specific, but always showing a degree of subjectivity. In this research, three assessment forms were used in the condition assessment of forty trees (ten by every forest species) in two green areas of Irati city. Through Friedman test it was noted significant difference among assessment forms used ( $\chi^{2} \mathrm{Fried}=69,194 ; \mathrm{GL}=2$; $\left.p=9,434^{-16}\right)$ to the valuation assigned to each tree. However, for better visual analysis of the general condition of urban trees, the assessment forms must contemplate the introduction of information about structural defects, condition of tree anchorage, existence of pests or diseases and the degree of risk. These adjustments become necessary and strategic to direct the attention of the evaluator for the main regions of assessment and to reduce subjectivity.
\end{abstract}

Keywords: Visual assessment, Vigor, Risk, Arboriculture. 


\section{INTRODUÇÃO}

No Brasil, os problemas decorrentes do crescimento de árvores nas calçadas, parques e praças, seja por danos a estruturas urbanas ou a pessoas, pouco despertaram o interesse por pesquisas relacionadas à avaliação da condição e estabilidade estrutural de árvores nos centros urbanos. Isso vem de encontro ao fato de que a falta de planejamento e cuidado para com as árvores urbanas tem promovido o aumento na queda de árvores ou a perda de vitalidade pelas condições adversas do ambiente urbano (SAMPAIO et al., 2010).

Por conta disso, conhecer o comportamento biomecânico e as condições onde pode ocorrer a ruptura do tronco e/ou ramos das árvores, especialmente frente a condições adversas de tempo, é de grande relevância nas áreas urbanas. Considera-se isso pela possibilidade de danos e riscos à integridade de propriedades e pessoas (JAMES; HARITOS; ADES, 2006; JIM; ZHANG, 2013), pois uma árvore pode ser considerada como de risco ou perigosa sempre que está estruturalmente debilitada e se há um alvo em potencial (HARRIS; CLARK; MATHENY, 1999).

Mesmo com a avaliação visual e o manejo de árvores de risco como parte do processo de gestão da arborização urbana e do licenciamento ambiental nas prefeituras municipais, ainda faltam critérios para a determinação do risco de ruptura de uma árvore e qual o melhor manejo a ser adotado (BRAZOLIN et al., 2014).

O processo de avaliação de uma árvore envolve o exame da condição geral da mesma (fitossanitária e estrutural) e das condições locais do entorno (área permeável, fiação, edificações, etc), com ponderações sobre o nível de risco que possa existir pela queda eventual ou provável sobre pessoas, veículos ou propriedades (ALBERS; POKORNY; JOHNSON, 2003; SCHALLENBERGER et al., 2010). Segundo Gonçalves, Stringheta e Coelho (2007) a avaliação e a tomada de decisão para supressão de árvores urbanas com potencial de queda ou que oferecem riscos tem sido motivo de maior preocupação porque envolve o patrimônio e a vida de terceiros.

Avaliações criteriosas podem ser feitas por meio de protocolos detalhados como o apresentado por ISA (2013), mas também pela utilização de aparelhos que permitem conhecer o grau de biodeterioração interna do lenho como o tomógrafo de impulso e o resistógrafo (LIN et al., 2008; ROLLO et al. 2013; LI et al., 2014), os quais auxiliam a tomada de decisão no processo de gerenciamento de risco de árvores urbanas.

Em geral, os procedimentos adotados pelas prefeituras municipais para avaliação de árvores são essencialmente visuais e tomam por base o reconhecimento de defeitos estruturais e de problemas fitossanitários presentes nas árvores. Entretanto, para diminuir a subjetividade da avaliação visual pode-se lançar mão de protocolos de avaliação que visam estabelecer um 
roteiro mínimo de observação e análise ao concentrar a avaliação nos fatores que interferem na estabilidade estrutural e no risco que uma árvore pode gerar.

Neste sentido, protocolos de avaliação já foram elaborados para auxiliar a análise da condição de árvores brasileiras (GONÇALVES; STRINGHETA; COELHO, 2007; SAMPAIO et al., 2010; SCHALLENBERGER et al., 2010), alguns exigindo maior atenção a detalhes e descrição de informação do que outros.

Kontogianni, Tsitsoni e Goudelis (2011) avaliaram a estabilidade de árvores tomando por base as características silviculturais de quatro espécies florestais, ao estudar a arborização de ruas da cidade de Thessaloniki, na Grécia. Estes autores propuseram a criação de um índice empírico que correlaciona a estabilidade das árvores com características silviculturais como o raio de projeção da copa, o índice de assimetria da copa e a altura da árvore. Esta proposição foi respaldada na argumentação de que quando as árvores estão sadias, sem qualquer sinal de declínio, e quando a velocidade dos ventos é menor do que $108 \mathrm{~km} / \mathrm{h}$ (limite acima do qual se caracteriza o principal motivo de ruptura e queda das árvores) as características silviculturais das árvores assumem maior importância na estabilidade das mesmas.

Diante do exposto, o objetivo geral desta pesquisa foi analisar e comparar a performance de diferentes protocolos de avaliação da condição geral de árvores componentes do tratamento paisagístico de diferentes tipologias da floresta urbana na cidade de Irati-PR.

\section{MATERIAL E MÉTODOS}

\section{Localização da área de estudo}

A presente pesquisa foi realizada na cidade de Irati, a qual está localizada na região Centro-Sul do Estado do Paraná entre as coordenadas 25 27' 56" S e 50 $37^{\prime \prime}$ '51" O (SCHALLENBERGER et al., 2010). Esta cidade, localizada no segundo planalto paranaense, próxima à Serra da Esperança, está inserida dentro do Ecossistema da Floresta Ombrófila Mista, Bioma Mata Atlântica.

Segundo a classificação climática de Köppen, o clima da região onde incide o município é do tipo Cfb (clima temperado), o qual corresponde à ocorrência de verões amenos e invernos com geadas severas e frequentes, não havendo estação seca definida. A precipitação média anual fica entre $1.600-1800 \mathrm{~mm}$ e a temperatura média anual entre $17-18^{\circ} \mathrm{C}$.

Os locais de coleta dos dados correspondem a duas tipologias de área verde componentes da floresta urbana da cidade: o Parque Aquático e de Exposições Santa Terezinha e a Praça Etelvina Andrade Gomes. 


\section{Procedimento de coleta dos dados}

Ao todo foram amostradas aleatoriamente 40 árvores, sendo 10 árvores de cada uma das principais espécies componentes das áreas verdes da cidade, o que corresponde a 6,5\% do número total apontado no levantamento de Schallenberger et al. (2010). As espécies escolhidas foram: Platanus $\mathrm{x}$ acerifolia (Aiton) Willd. (Platanaceae), Cryptomeria japonica (Thunb. ex L. f.) D. Don. (Cupressaceae), Tipuana tipu (Benth.) Kuntze (Fabaceae) e Populus deltoides W. Bartram ex Marshall (Salicaceae).

As variáveis mensuradas em cada indivíduo foram circunferência à altura do peito (CAP), altura total, altura de copa e quatro raios de projeção da copa, de acordo com a orientação dos pontos cardeais, para estimativa do diâmetro médio de copa.

Para cada indivíduo foi realizada avaliação da condição geral (estabilidade e risco de queda) de acordo com os índices e protocolos desenvolvidos por Gonçalves, Stringheta e Coelho (2007), Schallenberger et al. (2010) e Kontogianni, Tsitsoni e Goudelis (2011). Estes protocolos foram escolhidos devido à simplicidade e facilidade para execução da avaliação.

\section{Procedimento de análise dos dados}

Cada árvore amostrada foi avaliada de acordo com o roteiro de informações específicas de cada protocolo para avaliação da condição geral, visando a tomada de decisão sobre a manutenção ou remoção da árvore.

Por meio de cada protocolo foram valoradas as árvores, conforme escala sugerida, sendo possível enquadrá-las em classes de estabilidade e risco conforme a concepção de cada roteiro de avaliação. Por consequência, cada espécie teve uma amplitude particular de enquadramento nas classes consideradas, conforme cada protocolo.

Para realizar a análise comparativa foi necessária a divisão da amplitude de valores de cada protocolo em um mesmo número de classes de condição geral, sendo adotada a divisão proposta por Schallenberger et al. (2010): Classe 1: Péssima; Classe 2: Ruim; Classe 3: Regular; Classe 4: Boa; Classe 5: Excelente. Os protocolos de Schallenberger et al. (2010) e Kontogianni, Tsitsoni e Goudelis (2011) apresentavam uma mesma amplitude de valores, entre seis e vinte e quatro pontos, o que possibilitou adotar o mesmo padrão de divisão em classes de condição.

Por outro lado, o protocolo de Gonçalves, Stringheta e Coelho (2007) possuía treze classes de enquadramento das árvores selecionadas, sendo necessário agrupá-las para atender ao mesmo padrão de divisão em classes adotado por Schallenberger et al. (2010). Para isso procedeu-se ao agrupamento das cinco classes centrais, ao agrupamento de três classes intermediárias, em ambos os lados da escala sugerida pelos autores, e à 
individualização das classes dos extremos da escala. Com os cinco grupos de classe de condição formados adotou-se o critério de qualificação das classes de condição acima indicado.

Em cada protocolo, cada árvore recebeu um valor de condição geral a partir do qual foi possível enquadrá-la em uma das cinco classes de condição. A partir disso, os protocolos de avaliação foram comparados por meio de análise não paramétrica, com o Teste de Friedman ao nível de $1 \%$ de probabilidade, via software $\mathrm{R}$, a fim de verificar diferenças significativas de qualificação entre os mesmos.

\section{RESULTADOS E DISCUSSÃO}

As árvores avaliadas apresentaram características dimensionais distintas, concernentes ao modelo de crescimento assumido por cada uma das espécies amostradas (TABELA 1).

Tabela 1. Estatística descritiva das variáveis mensuradas nas árvores utilizadas para avaliação da condição geral

Table 1. Descriptive statistics of measured variables on trees used for the assessment of general condition

\begin{tabular}{|l|c|c|c|c|}
\hline \multirow{2}{*}{ Espécie } & \multicolumn{4}{|c|}{ CAP (m) } \\
\cline { 2 - 5 } & mín & méd & máx & CV\% \\
\hline Cryptomeria japonica & 0,62 & 0,81 & 1,03 & 15,08 \\
\hline Platanus $x$ acerifolia & 0,76 & 1,14 & 1,39 & 16,94 \\
\hline Populus deltoides & 0,88 & 1,03 & 1,37 & 12,26 \\
\hline Tipuana tipu & 0,93 & 1,23 & 1,77 & 21,81 \\
\hline \multirow{2}{*}{ Espécie } & \multicolumn{5}{|c|}{ Altura total (m) } \\
\cline { 2 - 5 } & mín & méd & máx & CV\% \\
\hline Cryptomeria japonica & 7,5 & 9,85 & 18,0 & 30,32 \\
\hline Platanus $x$ acerifolia & 6,5 & 11,7 & 14,0 & 19,45 \\
\hline Populus deltoides & 13,0 & 14,75 & 16,5 & 8,18 \\
\hline Tipuana tipu & 7,0 & 10,6 & 14,5 & 26,19 \\
\hline \multirow{2}{*}{ Espécie } & \multicolumn{5}{|c|}{ Altura de copa (m) } \\
\cline { 2 - 5 } & mín & méd & máx & CV\% \\
\hline Cryptomeria japonica & 5,0 & 6,9 & 12,0 & 27,92 \\
\hline Platanus $x$ acerifolia & 4,5 & 7,7 & 10,0 & 22,33 \\
\hline Populus deltoides & 8,0 & 9,85 & 12,0 & 13,33 \\
\hline Tipuana tipu & 4,0 & 5,3 & 6,5 & 14,88 \\
\hline Espécie & \multicolumn{5}{|c|}{ Diâmetro de Copa (m) } \\
\cline { 2 - 5 } & mín & méd & máx & CV\% \\
\hline Cryptomeria japonica & 3,2 & 3,86 & 5,3 & 20,01 \\
\hline Platanus $x$ acerifolia & 6,6 & 1,03 & 12,15 & 18,26 \\
\hline Populus deltoides & 7,3 & 8,51 & 10,3 & 9,75 \\
\hline Tipuana tipu & 9,95 & 12,43 & 16,35 & 16,21 \\
\hline
\end{tabular}

A variabilidade observada para as variáveis CAP (circunferência à altura do peito) e altura de copa apresentou-se relativamente baixa quando comparada ao trabalho realizado por Bobrowski (2011) para a avaliação de árvores na arborização urbana. Isto se deve ao fato do 
menor número de indivíduos amostrados e por terem sido obtidas informações em apenas duas áreas verdes da cidade, o que tende a reduzir a amplitude das variáveis consideradas. Bobrowski, Biondi e Figueiredo Filho (2012) observaram um coeficiente de variação para o DAP igual a 74,6\% na segunda ocasião de medição de um inventário da arborização de ruas em Curitiba, para um total de 122 espécies diferentes e 4360 indivíduos amostrados.

Por outro lado, ao realizar a análise morfométrica de 18 indivíduos de Tipuana tipu na arborização de ruas de Curitiba, Bobrowski, Lima Neto e Biondi (2013) também observaram baixos coeficientes de variação para as variáveis DAP, altura total e altura de copa.

A baixa variabilidade dos dados desta pesquisa torna-se um fator interessante ao desenvolvimento de análises comparativas entre protocolos de avaliação da condição geral de árvores urbanas, pois restringe a variação da informação aos fatores inerentes a cada roteiro de avaliação.

Cada protocolo de avaliação da condição geral das árvores apresentou distribuição particular das classes de valoração atribuídas às 40 árvores avaliadas (Figura 1). Por meio do Teste de Friedman constatou-se que há diferença estatisticamente significativa entre as classes de condição atribuídas por cada protocolo de avaliação às quarenta árvores avaliadas $\left(\chi^{2}\right.$ Fried $\left.=69,194 ; \mathrm{GL}=2 ; p=9,434^{-16}\right)$.

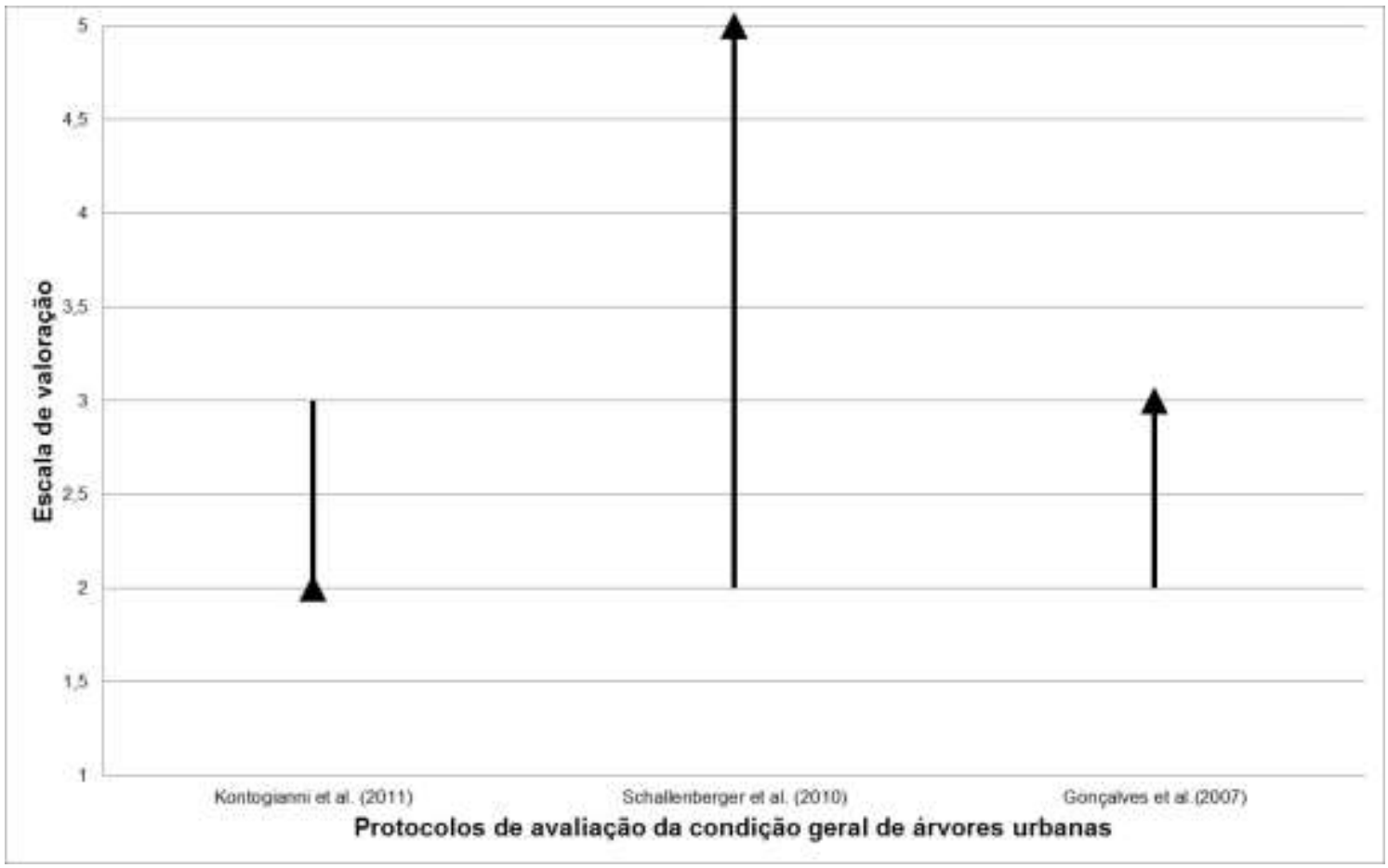

Figura 1. Amplitude de distribuição das notas atribuídas às árvores avaliadas por três protocolos diferentes. O símbolo em triângulo representa a mediana da classe de condição atribuída às árvores em cada protocolo

Figure 1. Distribution range of grades assigned to evaluated trees by three different protocols. The triangle symbol represents the median of the class condition attributed to trees in each protocol 
O protocolo de avaliação proposto por Kontogianni, Tsitsoni e Goudelis (2011) demonstrou tendência a qualificar as árvores como em condição geral ruim (classe 2). Isto se deve ao fato do protocolo conter um roteiro de avaliação baseado apenas em informações quantitativas (DAP, altura total, altura de copa, diâmetro de copa), de fácil predição, para expressar relações morfométricas interdimensionais das árvores e determinar a classe de condição geral a que pertence um indivíduo. Por mais que a árvore não apresente qualquer problema estrutural e/ou fitossanitário aparente ela pode ser qualificada como possuindo condição geral ruim, dependendo das suas características dimensionais (FIGURA 2) de altura total, proporção de copa, grau de esbeltez e índice de abrangência. Para estes autores, toda característica silvicultural/dimensional de uma árvore exerce influência sobre sua estabilidade.

\begin{tabular}{|c|c|}
\hline \multirow{3}{*}{$\begin{array}{c}\text { Árvore } 1 \\
\text { Platanus acerifolia }\end{array}$} & $\begin{array}{c}\text { Kontogianni et al. (2011) } \\
\text { Classe } 2\end{array}$ \\
\hline & $\begin{array}{c}\text { Schallenberger et al. (2010) } \\
\text { Classe } 5\end{array}$ \\
\hline & $\begin{array}{c}\text { Gonçalves et al. (2007) } \\
\text { Classe } 3\end{array}$ \\
\hline \multirow{3}{*}{$\begin{array}{c}\text { Árvore } 2 \\
\text { Populus deltoides }\end{array}$} & $\begin{array}{c}\text { Kontogianni et al. (2011) } \\
\text { Classe } 2\end{array}$ \\
\hline & $\begin{array}{c}\text { Schallenberger et al. (2010) } \\
\text { Classe } 5\end{array}$ \\
\hline & $\begin{array}{c}\text { Gonçalves et al. (2007) } \\
\text { Classe } 3\end{array}$ \\
\hline \multirow{3}{*}{$\begin{array}{c}\text { Árvore } 3 \\
\text { Tipuana tipu }\end{array}$} & $\begin{array}{c}\text { Kontogianni et al. (2011) } \\
\text { Classe } 2\end{array}$ \\
\hline & $\begin{array}{c}\text { Schallenberger et al. (2010) } \\
\text { Classe } 5\end{array}$ \\
\hline & $\begin{array}{c}\text { Gonçalves et al. (2007) } \\
\text { Classe } 3\end{array}$ \\
\hline
\end{tabular}

Figura 2. Exemplos da discrepância de qualificação em classes de condição atribuídas a algumas árvores avaliadas

Figure 2. Examples of qualifying discrepancy in condition classes assigned to some evaluated trees

O protocolo de avaliação proposto por Schallenberger et al. (2010) apresenta um roteiro mais detalhado de informações, em relação ao demais protocolos, mas mesmo assim possui uma escala subjetiva de decisão. Ao solicitar do avaliador a verificação da condição do tronco, da vitalidade da árvore e da existência de pragas ou doenças o roteiro proposto busca 
informações relevantes para a tomada de decisão, pois se uma árvore está debilitada por danos no tronco ou por pragas na copa, como a erva-de-passarinho, a estabilidade do indivíduo arbóreo pode estar comprometida e o grau de risco de queda acentuado. Diante disso, pode-se priorizar ações como a poda ou o corte da árvore, a fim de reduzir ou eliminar o risco envolvido. Devido às características mais abrangentes do protocolo, priorizando situações e regiões importantes nas árvores, os indivíduos arbóreos avaliados apresentaram melhor qualificação na escala de condição geral, fato este mais condizente com a realidade apresentada pelas árvores durante a inspeção visual.

Apesar do protocolo de avaliação proposto por Gonçalves, Stringheta e Coelho (2007) apresentar um roteiro detalhado de informações, divididas em categorias de avaliação (paisagística, ecológica, fitossanitária e riscos), o mesmo não conseguiu expressar adequadamente a condição geral das árvores avaliadas, conforme observações visuais, por atribuir um mesmo peso às diferentes categorias de informação. Entretanto, os autores afirmam que as características que colocam em risco a vida de pessoas tem peso decisivo na avaliação, mesmo que alguns critérios do método apresentem caráter emocional, influenciando a decisão de maneira mais subjetiva. Uma possível aplicação deste método seria utilizá-lo de forma complementar a outro, com atribuição de pesos entre as categorias de avaliação, pois uma árvore presente em um parque pode ter valoração diferente de uma árvore na arborização de ruas, mesmo apresentando tamanho e condição estrutural e fitossanitária aproximadas.

Nestes três protocolos de avaliação não são contempladas informações criteriosas a respeito de defeitos estruturais como rachaduras, podridões, declínio de copa, inclinação do tronco, brotações epicórmicas, dentre outros. Tampouco são solicitadas informações a respeito do grau de risco oferecido pela árvore, a partir de avaliações da condição do ancoramento da árvore ao solo para verificação de raízes de sustentação cortadas e/ou ocorrência de soerguimento do solo por podridão avançada das raízes e desequilíbrio da árvore por excesso de peso.

Para Elisson (2005) a determinação da probabilidade de queda, do impacto potencial das árvores e dos alvos que podem ser atingidos permite ao arborista avaliador ponderar com acurácia o risco de queda em diferentes situações, principalmente dos riscos inaceitáveis e dos elementos envolvidos no risco de queda da árvore. Neste sentido, Albers, Pokorny e Johnson (2003) afirmam que os gestores públicos têm a responsabilidade de criar e manter a floresta urbana utilizável pela população e que os silvicultores urbanos precisam possuir treinamento e experiência no reconhecimento de diferentes graus de risco, manejando a floresta urbana para níveis de risco aceitáveis.

Por mais subjetiva que seja a avaliação visual de árvores na floresta urbana, para determinação de sua condição geral e do grau de risco, a consideração sobre a avaliação de 
pontos estratégicos na árvore que sejam responsáveis pelos principais pontos de apoio estrutural são de extrema importância. Neste caso, a base do tronco e o entorno imediato, incluindo o solo e raízes de sustentação, bem como a base da copa ou ponto de inserção dos galhos da copa, devem ser assumidos como as principais regiões de avaliação devido ao maior suporte mecânico contra a ação do peso da árvore e de forças de tração e arraste pelo vento. Qualquer defeito estrutural nestes dois pontos pode induzir à queda da árvore ou de galhos grandes, aumentando o grau de risco oferecido pelo indivíduo arbóreo. O protocolo de avaliação proposto pela Sociedade Internacional de Arboricultura (ISA, 2013) é uma alternativa com maior rigor de análise por contemplar os pontos destacados como importantes na avaliação visual de árvores.

A prática da avaliação da condição geral das árvores realizada por órgãos públicos como as prefeituras municipais, diante de um grande volume de atendimentos, deve ser pautada em um criterioso protocolo de avaliação que sirva como um roteiro estruturado e dinâmico para a tomada de decisão, além de se constituir em um documento público onde ficam registradas as ações deliberadas e tomadas. Por conta disso, tal protocolo deve contemplar a redução da subjetividade e o direcionamento da avaliação para os principais pontos de apoio estrutural, prioritariamente, além de outros que possam comprometer a estabilidade da árvore e a segurança do entorno imediato da árvore.

Um dos pontos secundários da avaliação deve ser a distribuição de cargas na árvore (JAMES; HARITOS; ADES, 2006; KONTOGIANNI; TSITSONI; GOUDELIS, 2011), principalmente devido à ação da carga dinâmica do vento, mas também derivada de seu peso natural e seu equilíbrio ou expressão da arquitetura típica.

Como a análise visual é apenas parte do processo de decisão sobre a estabilidade da árvore, a avaliação realizada por meio de equipamentos eletrônicos, como o tomógrafo de impulso e o resistógrafo, devem se tornar parte da rotina de avaliação de árvores pelos órgão públicos, a fim de servirem como peças-chave ao fornecimento de informações mais acuradas em situações onde há dúvida sobre a condição da árvore ou haja a necessidade de descrição detalhada sobre problemas estruturais, tais como em árvores declaradas imunes de corte, conflitos judiciais, representatividade do exemplar arbóreo, dentre outros.

\section{CONCLUSÕES}

Os protocolos de avaliação da condição geral de árvores urbanas analisados mostraram tendências de valoração diferentes, fato este preocupante se utilizados para tomar decisões a nível técnico em diferentes condições de tratamento paisagístico, com diferentes graus de risco e condições de manejo. 
Desta forma, depreende-se que há necessidade de criar/adaptar/padronizar protocolos de avaliação brasileiros que contemplem defeitos estruturais reconhecíveis visualmente e as principais situações de risco de forma que sirvam como documentos básicos de apoio à decisão das ações de manejo, reduzindo subjetividades.

\section{REFERÊNCIAS}

ALBERS, J. S.; POKORNY, D. D.; JOHNSON, G. R. How to detect and assess hazardous defects in trees. In: POKORNY, J. D (Org.). Urban tree risk management: a community guide to program design and implementation. St. Paul: USDA, 2003. p. 41-116.

BOBROWSKI, R. Estrutura e dinâmica da arborização de ruas de Curitiba, Paraná, no período 1984 - 2010. 144 f. Dissertação (Mestrado em Engenharia Florestal) - Setor de Ciências Agrárias, Universidade Federal do Paraná, Curitiba, 2011.

BOBROWSKI, R.; BIONDI, D.; FIGUEIREDO FILHO, A. Dinâmica da distribuição diamétrica na arborização de ruas da cidade de Curitiba, Paraná, Brasil. Scientia Forestalis, Piracicaba, v.40, n.94, p.167-178, 2012.

BOBROWSKI, R.; LIMA NETO, E. M.; BIONDI, D. Alterações na arquitetura típica de Tipuana tipu (Benth.) O. Kuntze na arborização de ruas de Curitiba, Paraná. Ciência Florestal, Santa Maria, v.23, n.3, p.281-289, 2013.

BRAZOLIN, S.; TOMAZELLO FILHO, M.; YOJO, T.; OLIVEIRA NETO, N. A.; ALBUQUERQUE, A. R.; SETTE JÚNIOR. C. R. Propriedades físico-mecânicas do lenho deteriorado por fungos apodrecedores de árvores de Tipuana tipu. Cerne, Lavras, v.20, n.2, p.183-190, 2014.

ELLISON, M. J. Quantified tree risk assessment used in the management of amenity trees. Arboriculture \& Urban Forestry, Champaign, v.31, n.2, p.57-65, 2005.

GONÇALVES, W.; STRINGHETA, A. C. O.; COELHO, L. L. Análise de árvores urbanas para fins de supressão. Revista da Sociedade Brasileira de Arborização Urbana, Piracicaba, v.2, n.4, p.1-19, 2007.

HARRIS, R. W.; CLARK, J. R.; MATHENY, N. P. Arboriculture: integrated management of landscape trees, shrubs and vines. 3. ed. New Jersey: Prentice Hall, 1999.

ISA - INTERNATIONAL SOCIETY OF ARBORICULTURE. Tree Risk Assessment Manual. Champaign: ISA, 2013.

JAMES, K. R.; HARITOS, N.; ADES, P. K. Mechanical stability of trees under dynamic loads. American Journal of Botany, St. Louis, v.93, n.10, p.1522-1530, 2006.

JIM, C. Y.; ZHANG, H. Defect-disorder and risk assessment of heritage trees in urban Hong Kong. Urban Forestry \& Urban Greening, Amsterdam, v.12, n.4, 585-596, 2013.

KONTOGIANNI, A.; TSITSONI, T.; GOUDELIS, G. An index based on silvicultural knowledge for tree stability assessment and improved ecological function in urban ecosystems. Ecological Engineering, Amsterdam, v.37, n.6, p.914-919, 2011. 
LI, G.; WANG, X.; FENG, H.; WIEDENBECK, .; ROSS, R. J. Analysis of wave velocity patterns in black cherry trees and its effect on internal decay detection. Computers and Electronics in Agriculture, Amsterdam, v.10, p.32-39, 2014.

LIN, C.; KAO, Y.; LIN, T.; TSAI, M.; WANG, S.; LIN, L.; WANG, Y.; CHAN, M. Application of an ultrasonic tomographic technique for detecting defects in standing trees. International Biodeterioration \& Biodegradation, Amsterdam, v.62, p.434-441, 2008.

ROLLO, F. M. A.; SOAVE JUNIOR, M. A.; VIANA, S. M.; ROLLO, L. C. P.; COUTO. H. T. Z.; SILVA FILHO, D. F. Comparação entre leituras de resistógrafo e imagens tomográficas na avaliação interna de troncos de árvores. Cerne, Lavras, v.19, n.2, p. 331-337, 2013.

SAMPAIO, A. C. F.; DUARTE, F. G.; SILVA, E. G. C.; DE ANGELIS, B. L. D.; BLUM, C. T. Avaliação de árvores de risco na arborização de vias públicas de Nova Olímpia, Paraná. Revista da Sociedade Brasileira de Arborização Urbana, Piracicaba, v.5, n.2, p.82-104, 2010.

SCHALLENBERGER, L. S.; ARAUJO, A. J.; ARAUJO, M. N.; DEINER, L. J.; MACHADO, G. O. Avaliação de árvores urbanas nos principais parques e praças do município de Irati-PR. Revista da Sociedade Brasileira de Arborização Urbana, Piracicaba, v.5, n.2, p.105-123, 2010. 